\title{
Dolor Abdominal persistente
}

\author{
Beatriz Valero Serranoa, Pablo Franquelo Moralesa, \\ Juan Ramón Toledo Gómez ${ }^{\mathrm{a}}$, Pedro Fernández Iglesias ${ }^{\mathrm{b}}$
}

\begin{abstract}
a Servicio de Urgencias, Hospital Virgen de la Luz, Cuenca.

b Servicio de Radiodiagnóstico, Hospital Virgen de la Luz, Cuenca.

Correspondencia: Beatriz Valero Serrano, Servicio de Urgencias, Hospital Virgen de la Luz, C/ Hermandad de Donantes de Sangre $n^{\circ} 2,16002$ - Cuenca, España. E-mail: bvaleroserrano@gmail.com
\end{abstract}

Recibido el 12 de febrero de 2011.

Aceptado para su publicación el 4 de abril de 2011.

\section{RESUMEN}

El dolor abdominal es un motivo frecuente de consulta en atención primaria y urgencias, y constituye el síntoma más común en patología digestiva. La invaginación intestinal consiste en la introducción de una porción de intestino en otro distal y constituye una causa poco habitual de dolor abdominal en adultos, no obstante supone la causa mas frecuente de obstrucción intestinal en lactantes y niños menores de 5 años.

Se presenta el caso de una mujer de 14 años con dolor abdominal persistente por invaginación intestinal.

Palabras clave. Invaginación Intestinal, Obstrucción intestinal, Dolor Abdominal.

\section{ABSTRACT}

Persistent abdominal pain

Abdominal pain is a frequent complain in primary care and emergency departments, and is the most common symptom in gastrointestinal disorders. Intussusception occurs when a segment of bowel invaginates into the distal bowel and it is not a common cause of abdominal pain in adults, however, it is the most common cause of intestinal obstruction in infants and children under 5 years. We report a case of a fourteen year old girl with persistent abdominal pain due to intussusception.

Key words. Intussusception, Intestinal Obstruction, Abdominal Pain.

\section{INTRODUCCIÓN}

El dolor abdominal (DA) constituye un motivo habitual de consulta, tanto en los centros de atención primaria como en los servicios de urgencias hospitalarias.

EI DAes un síntoma frecuente con un elevado número de causas responsables del mismo, y su carácter normalmente inespecífico lo convierte en ocasiones en un desafío diagnóstico, de hecho hasta un $40-50 \%$ permanece sin un diagnóstico etiológico inicial. La intususcepción o invaginación intestinal cursa habitualmente con un DA intenso e intermitente como síntoma principal ${ }^{1}$, y constituye el motivo más frecuente de obstrucción intestinal en lactantes y niños menores de 5 años. No obstante, constituye una causa poco habitual de DA en adultos y en niños mayores.

Presentamos el caso de una mujer de 14 años con DA persistente y normalidad en las pruebas complementarias como consecuencia de un proceso de invaginación intestinal que se resolvió espontáneamente.

\section{OBSERVACIONES CLÍNICAS}

Mujer de 14 años que acudió a Urgencias por DA de carácter constante de 5 días de evolución, sin náuseas, vómitos, fiebre, alteración del hábito intestinal ni síndrome miccional asociados. Antecedentes personales de mononucleosis infecciosa hacía 2 años, sin ningún otro antecedente de interés. Había sido valorada dos días antes en su centro de salud por DA y disuria con diagnóstico de infección de tracto urinario inferior, por lo que seguía un tratamiento con amoxicilina, desapareciendo los síntomas de irritación vesical iniciales, pero con persistencia del DA. En la exploración física mostraba un buen estado general con TA de $120 / 70 \mathrm{~mm} / \mathrm{Hg}$ y $\mathrm{T}^{\mathrm{a}} 36.5^{\circ} \mathrm{C}$. A la 
palpación el abdomen era blando y depresible, con dolor en vacío e hipocondrio izquierdos. Sin masas ni megalias, ni signos de irritación peritoneal. Resto sin interés. Se realizó una analítica completa con hemograma, bioquímica, coagulación y sistemático de orina que resultaron normales. En la radiografía $(R x)$ de tórax y de abdomen no se encontró ningún hallazgo patológico. Ante la persistencia del dolor y la normalidad en las pruebas complementarias, se recurrió a una ecografía (ECO) abdominal, con presencia de adenopatías mesentéricas, estableciéndose posteriormente el diagnóstico diferencial con las posibles causas de un DA de fosa iliaca izquierda en una mujer (tabla 1$)^{2}$.

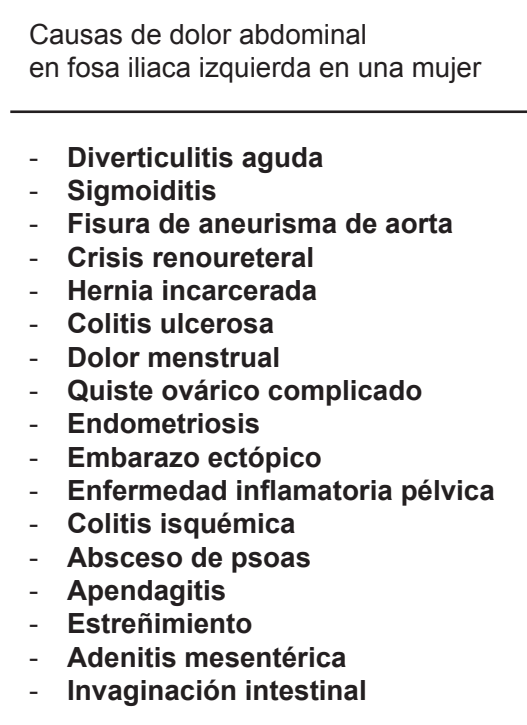

Tabla 1. Causas de dolor abdominal en fosa iliaca izquierda en una mujer ${ }^{2}$.
Por la intensidad, localización y persistencia del dolor, la ausencia de fiebre y edad de la paciente, el diagnóstico de adenitis mesentérica no parecía adecuado, por lo que se solicitó una tomografía

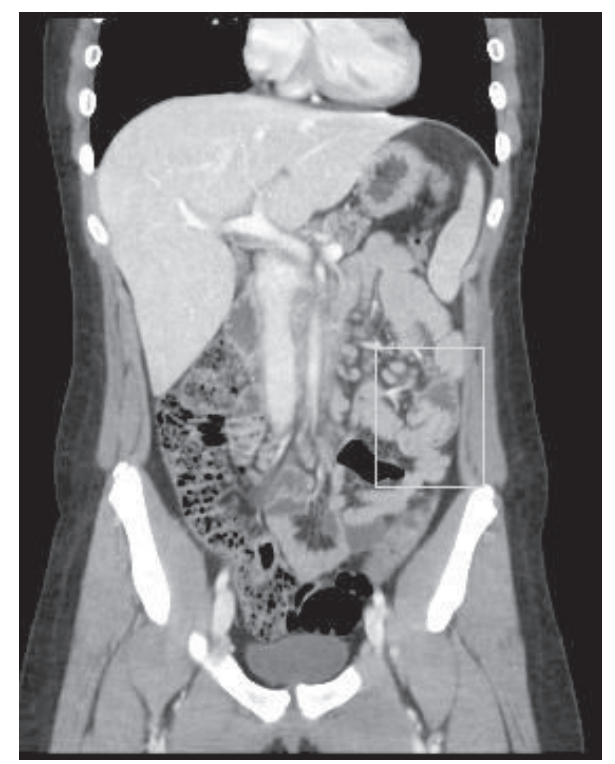

Figura 1. TC abdominal con corte longitudinal que muestra imagen en forma de "salchicha" o "pseudo riñón". computarizada (TC) abdominal, en la que se apreciaban múltiples adenopatías mesentéricas en vacío izquierdo con imagen sugestiva de una invaginación intestinal (figuras 1 y 2).

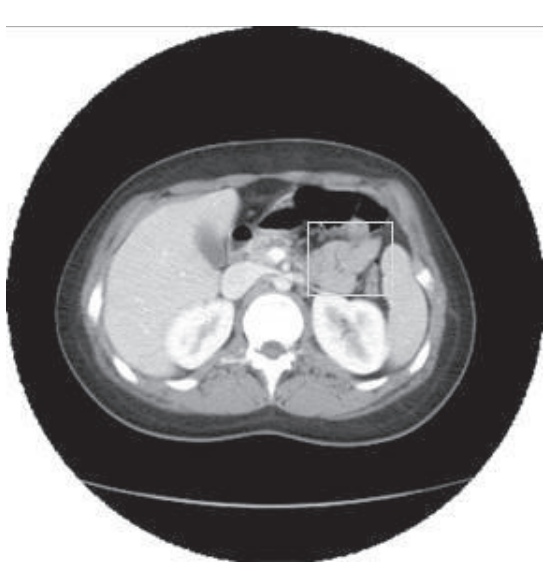

Figura 2. TC abdominal con corte trans-versal en la que se aprecia imagen en "diana". 
La paciente ingresó en el servicio de cirugía con diagnóstico de invaginación de intestino delgado (ID) ileo-ileal y, tras observación, analgesia y sueroterapia, presentó una mejoría clínica progresiva con desaparición del dolor e inicio de tolerancia oral sin incidencias. Se realizó ECO abdominal de control transcurridas 72 horas, sin hallazgos patológicos, por lo que fue dada de alta completamente asintomática.

\section{COMENTARIOS}

La invaginación intestinal es la introducción de una porción del intestino en otro generalmente distal, siguiendo los movimientos peristálticos del mismo. La localización más frecuente es en ID entre 75$80 \%$, con afectación del colon (forma ileo-cólica) o sin ella (formas ileo-iliales o yeyuno-yeyunales) ${ }^{3}$. Las presentaciones cólicas aisladas, colo-cólicas, y las ileoceco-cólicas son menos habituales.

El $80-90 \%$ tiene lugar en niños menores de un año y suelen ser primarias en su mayoría, originadas frecuentemente tras infecciones víricas que hipertrofian las placas de Peyer, abundantes en el íleon terminal, o bien pueden ser secundarias a procesos anatómicos o afecciones linfoides del intestino, hecho más habitual en niños mayores ${ }^{3-5}$. La etiología, clínica, diagnóstico y tratamiento difieren en la población pediátrica y adulta, de manera que en jóvenes y adultos se presenta como un proceso ciertamente inusual, no obstante en estudios recientes se han registrado hasta un $20 \%$ de invaginaciones en este rango de edad. En la edad adulta en un $80-90 \%$ presentan una lesión de base, pudiéndose tratar de procesos de carácter benigno como pólipos, lipomas, divertículo de Meckel, páncreas ectópico y adherencias, pero en ocasiones se originan por procesos malignos, afectando al colon fundamentalmente ${ }^{3,6}$.

En la edad pediátrica suele cursar con la triada clásica de DA de aparición brusca y carácter cólico, una masa abdominal palpable y la presencia de heces sanguinolentas en "jalea de grosella", sin embargo, en los adultos puede manifestarse de forma aguda como una obstrucción intestinal mecánica completa, o bien con una presentación subaguda o crónica, cursando con un DA intermitente que persiste durante meses o años, debido a que la invaginación es parcial o se reduce espontáneamente. Entre los síntomas más frecuentes en adultos, se encuentran el DA de carácter cólico, las náuseas y vómitos, así como ausencia de expulsión de gases y heces, aunque en ocasiones puede aparecer una diarrea sanguinolenta ${ }^{2,6}$.
Debido a la inespecificidad de la sintomatología, el diagnóstico habitualmente se realiza mediante una laparotomía exploradora urgente por una obstrucción, hemorragia o perforación intestinal.

La ECO y la TC abdominales son las pruebas radiológicas de mayor sensibilidad y especificidad, constituyendo esta última la técnica de elección ${ }^{7}$. La Rx simple de abdomen puede mostrar asas de ID dilatadas con niveles hidroaéreos y en los estudios baritados podemos observar un defecto de repleción a nivel de la invaginación ${ }^{3,8}$.

Aunque en algunos casos, como el nuestro, la reducción se produce de forma espontánea, el tratamiento habitual en los niños consiste en la reducción hidrostática o insuflación de aire, sin embargo, en adultos se recurre normalmente a la resección intestinal o de la lesión responsable de forma inicial, ya que casi siempre existe una causa orgánica de base.

Debido a las diferentes formas de presentación, etiología e implicaciones en el pronóstico, y teniendo en cuenta el aumento en la frecuencia observada en niños mayores y adultos, se debe considerar la invaginación como una causa potencial de obstrucción intestinal en todos los pacientes que consultan por DA agudo y persistente.

\section{BIBLIOGRAFÍA}

1. Guillén Paredes MP, Campillo Soto A, Martín Lorenzo JG Torralba Martínez JA, Mengual Ballester M, Cases Baldó MJ et al. Adult intussusception - 14 case reports and their outcomes. Rev Esp Enferm Dig. 2010; 102:32-40.

2. Santisteban López Y, Hermida Lazcano I, Albaladejo Ortiz C, Hernández Belmonte A. Dolor abdominal agudo. Medicine. 2007; 09:5657-64.

3. Mancebo L, Moral I, Castaño A, Sanz M J, Alegre N, Delgado M. Invaginación intestinal en el adulto. Emergencias. 2005; 17:87-90.

4. Martin-Lorenzo JG, Torralba-Martinez A, Liron-Ruiz R, Flores-Pastor B, Miguel-Perello J, Aguilar-Jimenez $\mathrm{J}$ et al. Intestinal invagination in adults: preoperative diagnosis and Management. Int J Colorectal Dis. 2004; 19:68-72.

5. Cochran AA, Higgins GL, Strout TD. Intussusception in traditional pediatric, nontraditional pediatric, and adult patients. Am J Emerg Med. 2010 Apr 1. [Epub ahead of print].

6. Calvo AM, Montón S, Rubio T, Repiso M, Sarasibar H. Invaginación intestinal en el adulto secundaria a lipoma yeyunal. An Sist Sanit Navar. 2005; 28:115-8.

7. Huang BY, Warshauer DM. Adult intussusception: diagnosis and clinical relevante. Radiol Clin N Am. 2003; 41:113751.

8. Bines JE, Ivanoff B, Justice F, Mulholland K. Clinical case definition for the diagnosis of acute intussusception. J Pediatr Gastroenterol Nutr. 2004; 39:511-8. 